The Astrophysical Journal, 552:L151-L154, 2001 May 10

(C) 2001. The American Astronomical Society. All rights reserved. Printed in U.S.A.

\title{
NEAR-INFRARED CORONAGRAPHIC IMAGING OF THE CIRCUMSTELLAR DISK AROUND TW HYDRAE
}

\author{
D. E. Trilling,${ }^{1,2}$ D. W. Koerner, ${ }^{1}$ J. W. Barnes, ${ }^{2,3}$ C. Ftaclas,${ }^{4}$ and R. H. Brown ${ }^{2,3}$ \\ Received 2001 January 2; accepted 2001 March 27; published 2001 April 25
}

\begin{abstract}
We present ground-based near-infrared ( $H$-band) imaging of the circumstellar disk around the nearby classical T Tauri star TW Hydrae. The scattered-light image shows a face-on disk with a radius of 4" (corresponding to 225 AU) and a morphology that agrees with recent images from the Hubble Space Telescope and the Very Large Array. The best-fit power-law for the disk's radial surface brightness profile obeys the law $r^{-3.3 \pm 0.3}$. We use our image and published continuum flux densities to derive properties of the disk with a simple model of emission from a flat disk. The best-fit values for disk mass and inner radius are $0.03 M_{\odot}$ and 0.3 AU, respectively; the best-fit values for the temperature, density, and grain opacity power-law exponents $(q, p$, and $\beta)$ are $0.7,1.3$, and 0.9 , respectively. These properties are similar to those of disks around classical T Tauri stars located in more distant molecular clouds. Because of TW Hydrae's nearby location and pole-on orientation, it is a uniquely favorable object for future studies of radial disk structure at the classical T Tauri stage.
\end{abstract}

Subject headings: accretion, accretion disks — circumstellar matter open clusters and associations: individual (TW Hydrae) -

planetary systems: protoplanetary disks — stars: individual (TW Hydrae) stars: pre-main-sequence

\section{INTRODUCTION}

The formation of the solar system from a nebula of gas and dust is strongly supported by observations of disks around young stars (Koerner 2001). Observations of these protoplanetary disks are largely confined to star-forming clouds at distances greater than $140 \mathrm{pc}$. In these regions, the majority of classical T Tauri stars (CTTSs) are found to have disks with masses in the range from $10^{-2}$ to $10^{-4} M_{\odot}$ (Beckwith et al. 1990; André \& Montmerle 1994). Aperture synthesis mapping of circumstellar gas has established that these disks have radii of a few hundred AU (Koerner \& Sargent 1995; Dutrey et al. 1996), in agreement with dimensions derived from Hubble Space Telescope (HST) images of young disks (O'Dell, Wen, \& Hu 1993; Padgett et al. 1999).

The discovery of a nearby lone CTTS by Rucinski \& Krautter (1983) affords a unique opportunity for high spatial resolution studies of a gas-rich disk. TW Hydrae (TW Hya; K7 Ve, $d \approx$ $56 \mathrm{pc}$ ) is the eponymous member of a nearby group of young stars (Kastner et al. 1997; Webb et al. 1999). The members of the TW Hydrae Association have ages of only a few to 10 million years despite their distance from a molecular cloud (Jensen, Cohen, \& Neuhäuser 1998; Webb et al. 1999). TW Hya (the star) stands in contrast to its namesakes by virtue of its large $\mathrm{H} \alpha$ equivalent width (220 ̊̊; Kastner et al. 1997), CO emission (Weintraub, Sandell, \& Duncan 1989; Zuckerman, Forveille, \& Kastner 1995; Kastner et al. 1997), and molecular hydrogen (Weintraub, Kastner, \& Bary 2000). Furthermore, the considerable 1.1 and $7 \mathrm{~mm}$ flux densities of 874 and $8 \mathrm{mJy}$, respectively (Weintraub et al. 1989; Wilner et al. 2000) — comparable, after distance scaling, to flux densities of CTTSs at much larger dis-

\footnotetext{
${ }^{1}$ University of Pennsylvania, David Rittenhouse Laboratory, 209 South 33d Street, Philadelphia, PA 19104; trilling@hep.upenn.edu.

${ }^{2}$ Visiting Astronomer at the Infrared Telescope Facility, which is operated by the University of Hawaii under contract to the National Aeronautics and Space Administration.

${ }^{3}$ Lunar and Planetary Laboratory, Space Sciences Building, P.O. Box 210092, University of Arizona, Tucson, AZ 85721-0092.

${ }^{4}$ Department of Physics, Michigan Technological University, 1400 Townsend Drive, Houghton, MI 49931.
}

tances-argue for an optically thick, massive $\left(\sim 10^{-2} M_{\odot}\right)$ circumstellar disk. This disk appears to be still actively accreting onto the star, as inferred from the $\mathrm{H} \alpha$ emission (Muzerolle et al. 2000). In short, TW Hya shows all the signs of being a CTTS with a surprisingly advanced age.

Recently, Krist et al. (2000, hereafter K00) and Weinberger et al. (1999) reported the detection of a face-on (radius $\approx 3.5^{\prime \prime}$ ) disk around TW Hya in HST images obtained with WFPC2 and NICMOS, respectively. Wilner et al. (2000) resolve (radius $\gtrsim 2^{\prime \prime}$ ) thermal emission from circumstellar dust around TW Hya with $7 \mathrm{~mm}$ VLA observations. In this Letter, we present the first ground-based, near-infrared image of the circumstellar disk around TW Hya and combine these data with thermal emission data to derive disk properties with a simple disk model. We find disk properties that are similar to those of disks around other CTTSs. The nearby TW Hya thus provides the bestknown opportunity to study the earliest stages of potentially planet-forming systems.

\section{OBSERVATIONS AND RESULTS}

We observed TW Hya on 2000 April 7 (UT) from NASA's Infrared Telescope Facility (IRTF) on Mauna Kea. We used CoCo, the Cold Coronagraph, to block the light from the central star and reduce the sidelobe intensity to allow imaging of the circumstellar disk; CoCo is used as a front end to the IRTF facility camera NSFCAM (Rayner et al. 1993; Shure et al. 1994). $\mathrm{CoCo}$ is a cryogenically cooled coronagraph with Gaussianapodized focal-plane masks and a cooled articulatable pupilplane mask (Wang et al. 1994; Toomey et al. 1998). We used a mask whose half-power (50\% transmission) diameter is 1 ".86. A series of eight exposures of TW Hya, typically $300 \mathrm{~s}$ long, were interspersed with similar ones of the nearby sky. The total onsource integration time was $2220 \mathrm{~s}$. We also obtained six $20 \mathrm{~s}$ exposures of the nearby G star SAO 202496 to measure the point-spread function (PSF); these observations were made immediately after the TW Hya observations. SAO 202496, which has a similar $H$ magnitude to TW Hya $(\Delta$ mag $\lesssim 0.5)$ and a sky separation of 6.4 , is well suited as a PSF comparison star for use in analysis of the TW Hya images. We observed both stars 

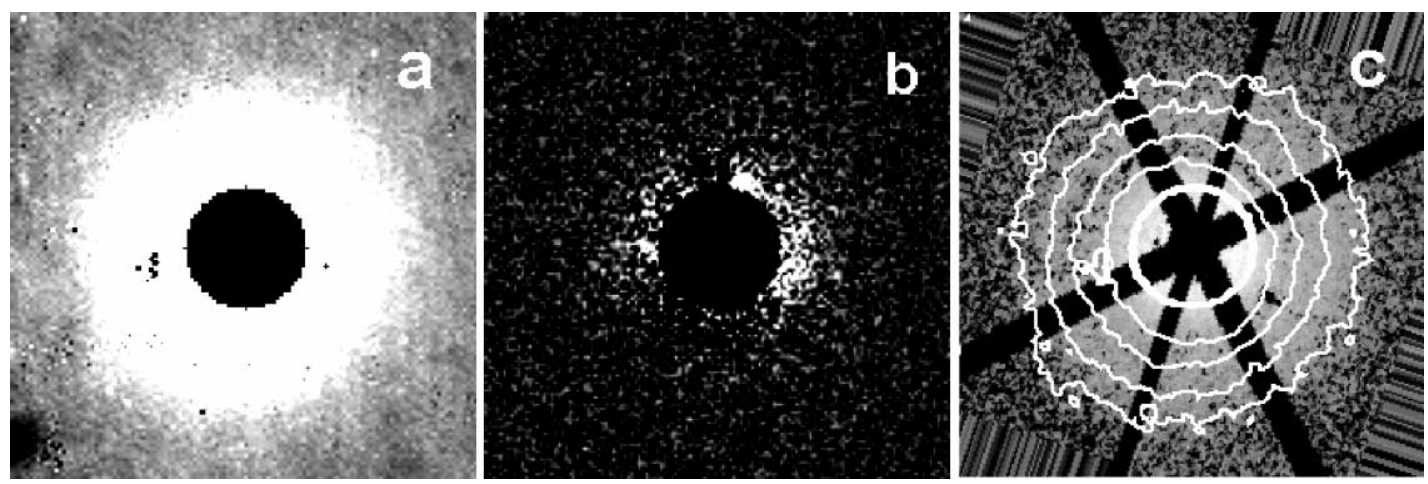

FIG. 1.-Images of the TW Hya disk and PSF-PSF residuals. (a) Our final (1620 s) image. This is a stack of six co-adds; each co-add is a 120 or $300 \mathrm{~s}$ TW Hya frame with a $20 \mathrm{~s}$ PSF star frame scaled, shifted, and subtracted from it. We have applied a linear stretch from 0 (black) to 0.67 (white) $\mathrm{mJy} \operatorname{arcsec}^{-2}$ to emphasize the outer radii. The central black circle represents our coronagraphic mask and was added in processing; its diameter is equal to the half-power point of the Gaussian focal-plane mask. The black circle in the lower left is a faint point source in the PSF frames; this point source is at least 10 mag fainter than the PSF star and is therefore completely negligible in the PSF subtractions. (b) Two $H$-band, $20 \mathrm{~s}$ PSF images subtracted from each other, illustrating typical residuals in the scaling and subtraction. The linear stretch in this panel is identical to that in (a). (c) I-band WFPC2 image (K00) with CoCo $H$-band contours (from panel $a$ ) overlaid. The WFPC2 data are shown in a logarithmic stretch (K00), and the CoCo contours correspond to $0.30,0.65,1.4$, and $3.0 \mathrm{mJy} \operatorname{arcsec}^{-2}$. The gray and black striped pattern near the edges of the rotated K00 image are artifacts of the rotation interpolation scheme we used; the original K00 image has north 18.5 counterclockwise from straight up (J. Krist 2001, private communication). All three panels have north up and east to the left. The field of view is approximately 7".04 on a side for each panel.

in $H$ band $\left(\lambda_{c}=1.62 \mu \mathrm{m}, \Delta \lambda=0.28 \mu \mathrm{m}\right)$; seeing was consistently 0.8 at air masses of 1.7-1.8 for TW Hya and 1.8 for SAO 202496. We also observed the photometric flux standard SAO 205948 (Elias et al. 1982) at similar air masses.

All exposures were sky-subtracted and flat-fielded. Each frame of SAO 202496 was aligned, scaled, and subtracted from each TW Hya frame. Alignment and scaling of the target and PSF frames were accomplished by minimizing the least-squares difference $\left(\chi^{2}\right)$ between target and PSF frames while varying amplitude scaling and position offsets. The least-squares difference was calculated in a region 4"-6".5 from the center of the frame. Image shifting in steps of 0.1 pixels was carried out in the Fourier domain. The amplitude was varied in step sizes corresponding to approximately $1 \%$ of the best-fit value. We carried out this same procedure on sets of two independent exposures of the PSF star to ensure that PSF-PSF results produced a null result and to characterize artifacts and residuals. Circular emission $3^{\prime \prime}-4$ " in radius was detected in all 48 combinations of target-PSF frames with a signal-to-noise ratio of several hundred compared with the background rms and 80 with respect to residuals in the PSF-PSF subtraction. Because CoCo rejects $90 \%-95 \%$ of the light from the central star, scattered light from the circumstellar disk itself dominates from 2" radius out to the edge of the disk (see below).

Figure $1 a$ shows our best image of the nebulosity around TW Hya. A circular halo of size $r=4^{\prime \prime}$ (5 $\sigma$ detection limit) is clearly apparent. The peak intensity of the emission is $6.8 \pm$ $1.1 \mathrm{mJy} \operatorname{arcsec}^{-2}$ at a radial distance of 0.94 from the star, the smallest radial distance we can see using this focal-plane mask. The reported uncertainty comprises a conservative estimate of errors. The largest source of error is the imperfect centering of the stars behind the coronagraphic mask. This effect results in azimuthal variations of approximately $15 \%$ in the final subtracted image when averaged over complete quadrants; this is estimated by assuming that the circumstellar emission is axisymmetric and centered on the star. By contrast, the $1 \sigma$ pixel-to-pixel rms noise in the image background is $10 \mu \mathrm{Jy} \operatorname{arcsec}^{-2}$, corresponding to an error of much less than $1 \%$. (This background noise is dominated by noise from the scaled PSF star background.) The conservative nature of our photometric error estimate is illustrated by the subtractions of independent observations of the PSF star
(Fig. 1b). Here we show the difference between two independent measurements of SAO 202496; the residuals have a maximum above-background emission at just over $1^{\prime \prime}$ with an average peak surface brightness of $0.1 \mathrm{mJy} \operatorname{arcsec}^{-2}$. These images are subject to the same centering errors as the PSF-subtracted image of TW Hya, and the residuals seen in the PSF-PSF image can represent the error due to imperfect centering. The total integrated flux density from the circumstellar emission around TW Hya is $21.6 \pm 3.5 \mathrm{mJy}$; by comparison, the total integrated flux density of the residuals in the PSF-PSF image is around $0.25 \mathrm{mJy}$. The fidelity of the final TW Hya image is bolstered by the comparison displayed in Figure 1c. Here we show the K00 WFPC2 I-band image in gray scale overlaid with contours from our TW Hya image (Fig. 1a). In radial extent and orientation, there is a very clear and convincing match between the two.

In Figure 2, we show azimuthally averaged radial profiles of TW Hya and PSF star images before PSF subtraction (thin solid lines) as well as the radial profile of the final, PSFsubtracted image of the circumstellar emission around TW Hya (thick solid line). We also show the azimuthally averaged radial profile of the PSF-PSF residual (thick dotted line). This figure illustrates a point made above: outside of $2^{\prime \prime}$, a circumstellar excess is seen in the TW Hya image compared with the PSF star image before PSF subtraction from either image. This excess extends to around 4" in the fully reduced (PSF-subtracted) data (Fig. 2, thick solid line), corresponding to an outer radius of $225 \mathrm{AU}$ at the distance of TW Hya. A single power-law fit to the radial profile follows the law $r^{-3.3 \pm 0.3}$ (Fig. 2, thick dashed line), although the data do not precisely follow a single power law (see Fig. 2). Although we find scant evidence for the multiple power laws derived by K00, we do not have the spatial resolution that $\mathrm{K} 00$ had, and our results are in good agreement with the average slope $(\sim-3.5)$ of the K00 broken power law. Our data may show that the radial profile of the disk becomes steeper outside of $200 \mathrm{AU}$, in rough agreement with K00; this level, however, is close to the background level.

\section{MODELING AND DISCUSSION}

The $1.1 \mathrm{~mm}$ continuum flux density from TW Hya $(874 \pm 54 \mathrm{mJy})$ implies a total circumstellar mass (gas + dust) 


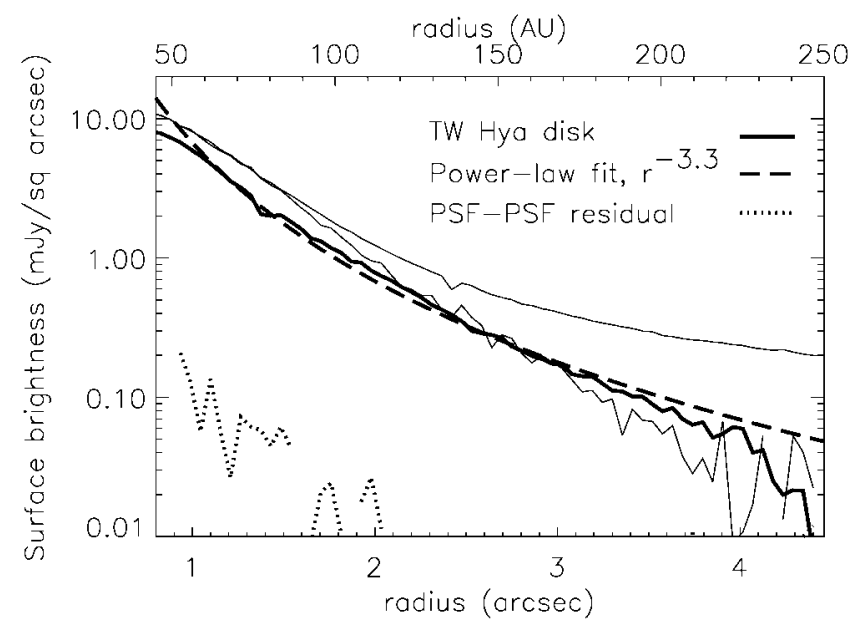

FIG. 2.-Azimuthally averaged radial profiles for images of TW Hya and the PSF star, both before PSF subtraction, and of the final (PSF-subtracted) TW Hya circumstellar disk. The upper and lower thin solid lines show radial profiles of images of TW Hya and the PSF star, respectively, before PSF subtraction. The PSF star profile is scaled to be equal to the TW Hya profile at 0 .'94. The TW Hya profile is distinctly different from the PSF profile outside of around 2" (110 AU), showing that we detect the circumstellar disk in the image of TW Hya before PSF subtraction. We also show (thick solid line) the radial profile of the final (PSF-subtracted) image of the circumstellar disk (the image shown in Fig. 1a). The best power-law fit to the disk, which follows an $r^{-3.3}$ power law, is shown as the thick dashed line. For comparison, the azimuthally averaged radial profile of the PSF-PSF residual (Fig. 1b) is shown in the thick dotted line; this residual is quite small compared with the TW Hya disk detection.

of order $10^{-2} M_{\odot}$ (Weintraub et al. 1989). For micron-sized grains, this implies a total dust grain cross-sectional area around $10^{7} \mathrm{AU}^{2}$, more than large enough to intercept all stellar light if oriented in a spherical shell with radius similar to the disk size. Additionally, the fractional disk luminosity is $\sim 0.3$ (Zuckerman et al. 1995), but the ratio of disk reflected light to stellar light is only $\sim 10^{-2}$ at the $H$ band. Both statements imply that the optical depth in the disk midplane must be quite high. This precludes the use of a model that derives temperature structure under the assumption of direct thermal equilibrium between stellar radiation and individual grains (e.g., Backman, Witteborn, \& Gillett 1992). Instead, the temperature structure may reflect a variety of effects, including viscous internal heating, reprocessing of stellar radiation, and superheating in a surface layer (e.g., Lynden-Bell \& Pringle 1974; Chiang \& Goldreich 1997). Without a priori knowledge of the relative magnitudes of these influences, we find it simplest to freely parameterize the temperature structure in a fit to the spectral energy distribution (SED) as in Beckwith et al. (1990).

We derive properties of the TW Hya circumstellar disk by using a simple, two-dimensional model that incorporates both our imaging data and the thermal continuum flux densities shown in Figure 3. Our best disk model is also shown in Figure 3. Following Beckwith et al. (1990), we assume power-law radial profiles for the temperature and surface density, $T=T_{1}(r)^{-q}$ and $\Sigma=\Sigma_{0}\left(r / r_{0}\right)^{-p}$, where $r$ is in astronomical units, $T_{1}$ is the temperature at $1 \mathrm{AU}$, and $\Sigma_{0}$ (the surface density at $r_{0}$ ) is couched in terms of the disk mass $M_{\text {disk }}$ by integrating over the disk surface area. The grain emissivity is $\kappa=0.1\left(\nu / 10^{12} \mathrm{~Hz}\right)^{\beta} \mathrm{cm}^{2} \mathrm{~g}^{-1} . T_{1}$ and $q$ are found to be $125 \mathrm{~K}$ and 0.7 , respectively, by fitting points at infrared wavelengths where the disk radiation is optically thick; $T_{1} \approx 125 \mathrm{~K}$ is similar to results for other CTTSs (Beckwith et al. 1990). An inner radius $R_{0}$ of $0.3 \mathrm{AU}$ is required

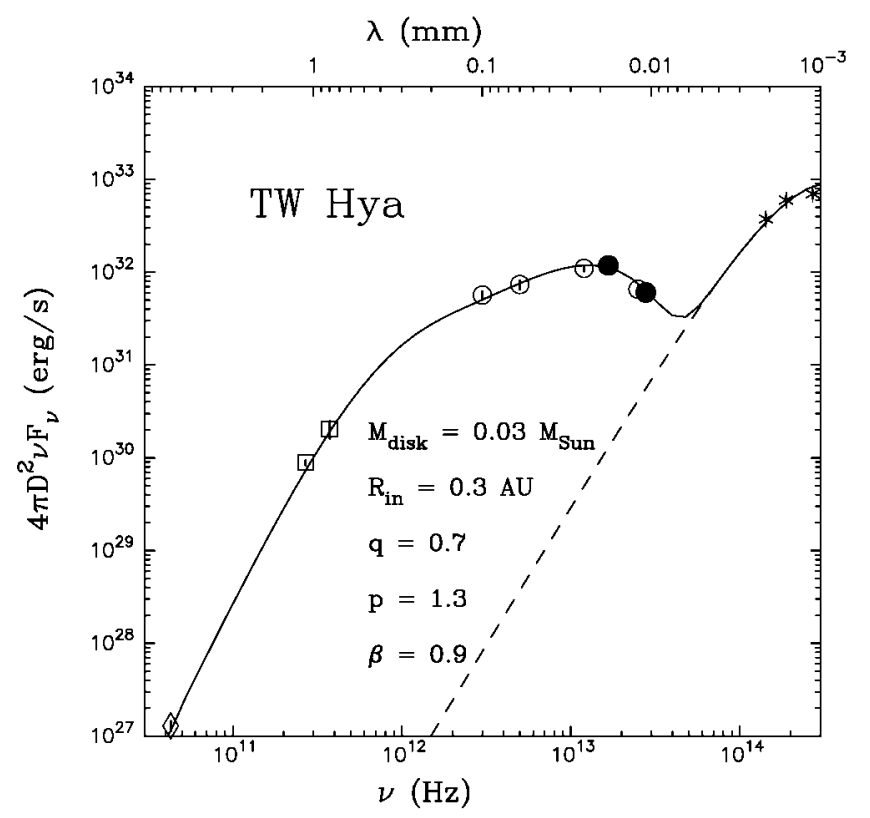

FIG. 3.-SED for TW Hya. The dashed line is a blackbody for the star TW Hya $(4080 \mathrm{~K})$. The solid line is the sum of stellar and disk fluxes, including the best-fit disk model found with the five shown parameters varied. All parameters except $p$ are well constrained by the multiwavelength fluxes and nearinfrared data. The asterisks are near-infrared $(J H K)$ fluxes from Webb et al. (1999); the filled circles are mid-infrared fluxes from Jayawardhana et al. (1999b); the open circles are IRAS fluxes; the squares are 0.8 and $1.1 \mathrm{~mm}$ data from Weintraub et al. (1989); and the triangle is the $7 \mathrm{~mm}$ flux density measurement from Wilner et al. (2000). Error bars are small vertical lines shown for each data point.

to fit the $12 \mu \mathrm{m}$ point. The face-on orientation and outer radius of $225 \mathrm{AU}$ are indicated by the image in Figure $1 a$. We vary the remaining parameters $\left(M_{\text {disk }}, p\right.$, and $\beta$ ) over a grid of appropriate values while minimizing the reduced $\chi^{2}$ difference between model and data. Our derived best-fit values are $M_{\text {disk }}=$ $0.03 M_{\odot}, p=1.3$, and $\beta=0.9$.

Typical $q$-values for CTTSs $(\sim 0.5$; Beckwith et al. 1990) can indicate back-warming from an envelope or reprocessing by a geometrically flared disk (Natta 1993; Chiang \& Goldreich 1997). Our larger value for $q(0.7)$ is closer to the value expected for either a standard viscous accretion or geometrically flat reprocessing disk, perhaps suggesting the disk flattening implied for some transitional sources (Miyake \& Nakagawa 1995). Radial brightness power-law exponents for geometrically flat to fully flared disks are expected to range from -3 to -1.8 . Our power-law fit exponent is $-3.3 \pm 0.3$, consistent with the average K00 exponent of $\sim-3.5$. Steeper indices than -3 might result from regions of the disk that are shadowed from the star. The higher angular resolution K00 observations yield regions with power-law exponents between -0.5 and -5.9 , perhaps suggesting the kind of ripples that can exist for passively heated disks (Chiang 2000; Dullemond 2000).

The inner disk hole $\left(R_{0}=0.3 \mathrm{AU}\right)$ implied by our model is required by the deficit of disk-derived flux at $12 \mu \mathrm{m}$ compared with that at $18 \mu \mathrm{m}$. More sophisticated models of disks-including geometric as well as advanced radiative transfer considerations - may show a relative decrease at $12 \mu \mathrm{m}$ without an inner disk hole (Chiang \& Goldreich 1997, 1999); our model simply requires an inner disk hole as a substitute for more complex calculations. Future work includes applying these more sophisticated models to our TW Hya data. 
We derive a temperature power-law index of $q=0.7$ from the disk continuum emission, consistent with the average K00 scattered-light disk scale height radial power-law exponent of 1.15 (Kenyon \& Hartmann 1987). Similarly, both we and K00 derive (average) density power-law indices of $p=1.3$. Our value for $p$ is not narrowly constrained, and values between 1.1 and 1.5 all provide acceptable fits to the continuum data; however, our $p$ is better constrained than is typical for SED fits since the principal degeneracy is with outer disk radius, which is given here by our image. K00 likewise found their density exponent not well constrained. A value of $p=0.75$ is the theoretical expectation for steady state accretion (LyndenBell \& Pringle 1974), although Bell et al. (1997) incorporate effects such as disk opacity and shadowing and find complicated radial density distributions with effective average $p$ closer to unity.

Our derived $M_{\text {disk }}$ of $0.03 M_{\odot}$ and $\beta$ of 0.9 are narrowly constrained by the long wavelength $(0.8,1.1$, and $7 \mathrm{~mm})$ data; Wilner et al. (2000) found $M_{\text {disk }}=0.03 M_{\odot}$ and $\beta=1.0$. These derived $\beta$-values differ from $\beta$ for the interstellar medium $(\sim 2)$, but agree with that found for dust around other CTTSs (Beckwith \& Sargent 1991; Dutrey et al. 1996). This effect has been explained as the result of aggregation of dust grains into larger disk particles (Beckwith, Henning, \& Nakagawa 2000).

The age derived for TW Hya, (3-10) $\times 10^{6} \mathrm{yr}$ (Jensen et al. 1998; Webb et al. 1999), is somewhat older than the ages typically reported for other CTTSs with similar disks, $10^{5}$ to $5 \times 10^{6} \mathrm{yr}$ (Beckwith et al. 1990). It is therefore surprising that TW Hya possesses youthful disk properties, even though it exhibits a stellar age at the upper end of this range and disks around other Association members are considerably more evolved than the TW Hya disk (Koerner et al. 1998; Jayawardhana et al. 1998, 1999a, 1999b; Schneider et al. 1999; Koerner et al. 2000). However, because there can exist a wide variation in initial disk masses and lifetimes (e.g., Osterloh \& Beckwith 1995) and because ages for CTTSs in star-forming regions have great uncertainties (due to poorly ascertained distances and extinction corrections), we suggest that disk properties should be taken as the key indicator of a disk evolutionary state that is only approximately correlated with H-R diagram-derived stellar ages. In this view, TW Hya is especially useful as the closest example of a protoplanetary disk in the classical $\mathrm{T}$ Tauri phase and with a mass similar to the minimum-mass solar nebula. Since disk properties at this stage are likely to hold great importance for theories about the origin of planetary systems, TW Hya's nearby location and face-on orientation make it ideal for future high-resolution studies of T Tauri disks.

We thank the IRTF day crew and telescope operators for their invaluable assistance. We thank Ray Jayawardhana for help with the planning, execution, and interpretation of these observations. We thank Zahed Wahhaj, Hubert Klahr, Eugene Chiang, and John Krist for useful conversations and two anonymous referees for their helpful comments. This work was supported in part by grants to Doug Lin and Peter Bodenheimer and NASA grant AST 96-18803 and NSF grant NAG5-6310 to $\mathrm{C}$. F.

\section{REFERENCES}

André, P., \& Montmerle, T. 1994, ApJ, 420, 837

Backman, D. E., Witteborn, F. C., \& Gillett, F. C. 1992, ApJ, 385, 670

Beckwith, S. V. W., Henning, T., \& Nakagawa, Y. 2000, in Protostars and Planets IV, ed. V. Mannings, A. P. Boss, \& S. S. Russell (Tucson: Univ. Arizona Press), 533

Beckwith, S. V. W., \& Sargent, A. I. 1991, ApJ, 381, 250

Beckwith, S. V. W., Sargent, A. I., Chini, R. S., \& Güsten, R. 1990, AJ, 99, 924

Bell, K. R., Cassen, P. M., Klahr, H. H., \& Henning, Th. 1997, ApJ, 486, 372

Chiang, E. I. 2000, Ph.D. thesis, Caltech

Chiang, E. I., \& Goldreich, P. 1997, ApJ, 490, 368 1999, ApJ, 519, 279

Dullemond, C. P. 2000, A\&A, 361, L17

Dutrey, A., Guilloteau, S., Duvert, G., Prato, L., Simon, M., Schuster, K., \& Ménard, F. 1996, A\&A, 309, 493

Elias, J. H., Frogel, J. A., Matthews, K., \& Neugebauer, G. 1982, AJ, 87, 1029

Jayawardhana, R., Fisher, S., Hartmann, L., Telesco, C., Piña, R., \& Fazio, G. 1998, ApJ, 503, L79

Jayawardhana, R., Hartmann, L., Fazio, G., Fisher, S., Telesco, C., \& Piña, R. 1999a, ApJ, 520, L41 1999b, ApJ, 521, L129

Jensen, E. L. N., Cohen, D. H., \& Neuhäuser, R. 1998, AJ, 116, 414

Kastner, J. H., Zuckerman, B., Weintraub, D. A., \& Forveille, T. 1997, Science, 277,67

Kenyon, S. J., \& Hartmann, L. 1987, ApJ, 323, 714

Koerner, D. W. 2001, in The 4th Teton Summer School Conf.: Galactic Structure, Stars, and the Interstellar Medium, ed. C. W. Woodward, M. Bicay, \& J. M. Schull (San Francisco: ASP), in press

Koerner, D. W., Jensen, E. L. N., Cruz, K. L., \& Gultekin, K. 2000, ApJ, 533, L37
Koerner, D. W., \& Sargent, A. I. 1995, AJ, 109, 2138

Koerner, D. W., Ressler, M. E., Werner, M. W., \& Backman, D. E. 1998, ApJ, 503, L83

Krist, J. E., Stapelfeldt, K. R., Ménard, F., Padgett, D. L., \& Burrows, C. J. 2000, ApJ, 538, 793 (K00)

Lynden-Bell, D., \& Pringle, J. E. 1974, MNRAS, 168, 603

Miyake, K., \& Nakagawa, Y. 1995, ApJ, 441, 361

Muzerolle, J., Calvet, N., Briceño, Hartmann, L., \& Hillenbrand, L. 2000, ApJ, 535, L47

Natta, A. 1993, ApJ, 412, 761

O’Dell, C. R., Wen, Z., \& Hu, X. 1993, ApJ, 410, 696

Osterloh, M., \& Beckwith, S. V. W. 1995, ApJ, 439, 288

Padgett, D. L., Brandner, W., Stapelfeldt, K. R., Strom, S. E., Terebey, S., \& Koerner, D. W. 1999, AJ, 117, 1490

Rayner, J. T., et al. 1993, Proc. SPIE, 1946, 490

Rucinski, S. M., \& Krautter, J. 1983, A\&A, 121, 217

Schneider, G., et al. 1999, ApJ, 513, L127

Shure, M., et al. 1994, Exp. Astron., 3, 239

Toomey, D. W., Ftaclas, C., Brown, R. H., \& Trilling, D. 1998, Proc. SPIE, 3354,782

Wang, S.-I., et al. 1994, Proc. SPIE, 2198, 578

Webb, R. A., Zuckerman, B., Platais, I., Patience, J., White, R. J., Schwartz, M. J., \& McCarthy, C. 1999, ApJ, 512, L63

Weinberger, A. J., Schneider, G., Becklin, E. E., Smith, B. A., \& Hines, D. C. 1999, BAAS, $194,69.04$

Weintraub, D. A., Kastner, J. H., \& Bary, J. S. 2000, ApJ, 541, 767

Weintraub, D. A., Sandell, G., \& Duncan, W. D. 1989, ApJ, 340, L69

Wilner, D. J., Ho, P. T. P., Kastner, J. H., \& Rodríguez, L. F. 2000, ApJ, 534, L101

Zuckerman, B., Forveille, T., \& Kastner, J. H. 1995, Nature, 373, 494 